\title{
Fibreoptic bronchoscopy and diagnosis of pulmonary lesions in lymphoma and leukaemia
}

\author{
M J PHILLIPS, R K KNIGHT, AND MALCOLM GREEN
}

From the Chest Department, St Bartholomew's Hospital, London

ABSTRACT Fifty-six fibreoptic bronchoscopies were performed on 42 patients with Hodgkin's disease, lymphoma, or leukaemia and pulmonary complications which did not respond to conventional antibiotics. All these patients had received chemotherapy, radiotherapy, or both for the treatment of their underlying conditions. Twenty-two bronchoscopic procedures were complicated by thrombocytopenia and neutropenia, requiring platelet transfusion before bronchoscopy, and many patients were hypoxaemic. Visual examination of the tracheobronchial tree, alveolar lavage, bronchial brushing, and transbronchial biopsy were carried out as approximate. Three patients had minor pulmonary haemorrhage, and three developed a pneumothorax after transbronchial biopsy. A specific diagnosis was obtained in 14 of 18 patients $(78 \%)$ with diffuse chest radiographic abnormalities, in seven of 11 patients $(64 \%)$ with lobar or segmental (focal) abnormalities, in two of eight patients with small (local) lesions, and in three of five patients with hilar abnormalities. In only three patients were infections diagnosed. It is concluded that fibreoptic bronchoscopy is a useful and safe diagnostic procedure in this situation but its value depends upon the type of radiological abnormality.

It has long been recognised that pulmonary lesions occur frequently in patients with Hodgkin's disease, malignant lymphoma, and leukaemias, and that there are many possible causes. Such pulmonary complications may be the result of infection, either by the common bacterial organisms, by Mycobacterium tuberculosis, or by a wide variety of opportunistic pathogens which may invade lung tissue in patients who are immunocompromised as a result of their disease, of chemotherapy, or of radiotherapy. ${ }^{1}$ The more common of these opportunistic pathogens include Pneumocystis carinii, Aspergillus, Nocardia, and certain viruses. Several surveys ${ }^{2} 3$ have shown that lung involvement in Hodgkin's disease is common, occurring in up to $43 \%$ of patients, and that in addition the pleura, mediastinum or hilar nodes may be affected. Lung involvement may also occur in malignant lymphomas and in leukaemias. ${ }^{45}$ Separate neoplastic diseases of the lung may also be found with increased frequency in this group of patients, especially when rendered immuno-deficient by cytotoxic agents or radiotherapy. ${ }^{6}$ Chemotherapy and radiotherapy may

Address for reprint requests: Dr M Green, Chest Department, St Bartholomew's Hospital, West Smithfield, London EC1A 7BE. themselves produce a pneumonitis with pulmonary shadowing, the signs and symptoms of which are indistinguishable from those resulting from infective causes. ${ }^{78}$ Finally it has been suggested ${ }^{9}$ that occult pulmonary haemorrhage is a more common cause of pulmonary infiltration in thrombocytopenic immuno-compromised patients than had been thought previously.

Pulmonary complications are a frequent cause of morbidity or death in this group of patients. A proportion of the causes are susceptible to treatment, and it is important to make a specific diagnosis because of the wide range of possible causes and the toxicity of many of the drugs used in their treatment. Clinical signs and symptoms and chest radiographic appearances have proved to be poor indicators of the cause of lung shadowing. Examination and culture of sputum and transtracheal aspirates may provide the diagnosis in the case of the more common bacterial pathogens, but are usually unhelpful for opportunistic infections or neoplastic processes, and have furnished no information in cases where pulmonary haemorrhage or a pneumonitis caused by radiation or chemotherapy has occurred. In these instances lung tissue is required for 
examination. Before the advent of fibreoptic bronchoscopy (FOB) the techniques used included needle aspiration, percutaneous ("closed") lung biopsy by cutting needles or trephine air drill, and open lung biopsy via a thoracotomy. ${ }^{10^{10-12}}$ All these techniques have a definite morbidity and even mortality. Many studies have shown FOB to be a useful and relatively safe technique in the diagnosis of pulmonary lesions, ${ }^{13-15}$ but very few patients with lymphoma or leukaemia have been studied using full bronchoscopic technique. ${ }^{16} \mathrm{We}$ describe an evaluation of FOB in a group of patients with Hodgkin's disease, lymphoma, or leukaemia, many of whom had risk factors present. All these patients had FOB, with regional alveolar lavage, endobronchial brushing, and transbronchial lung biopsies where appropriate.

\section{Methods}

Fibreoptic bronchoscopy was performed on 42 patients with Hodgkin's disease, lymphoma, or leukaemia, in whom various pulmonary complications had developed which did not respond to conventional antibiotic therapy (table 1). Many of these patients had received chemotherapy, radiotherapy, or both for the treatment of their underlying condition, and in a large proportion risk factors such as arterial hypoxaemia, thrombocytopenia, and neutropenia were present. There were 28 men and 15 women ranging in age from 17 to 68 years (average age $42 \mathrm{yr}$ ).

Patients were categorised according to chest radiographic changes which were designated as being diffuse-if more than one of the six zones in the chest film was involved, focal-if there was segmental or lobar involvement, local-if the lesion was $3 \mathrm{~cm}$ or less in diameter, or hilar-if the hilar region appeared prominent or enlarged.

Those patients who were pyrexial and had cough or sputum production associated with their pulmonary lesions were investigated first with culture of sputum, blood, and throat swabs. Blood was sent for viral, Mycoplasma, Aspergillus, Toxoplasma, and Cryptococcus serology and transtracheal aspirations were carried out in some. Normally these patients were given broad spectrum antibiotic therapy early as part of a "fever" regimen. If these measures failed to establish the diagnosis or to improve the clinical status of the patient, FOB was performed. Some patients who developed lesions on the chest radiograph unassociated with pyrexia or respiratory symptoms were referred directly for bronchoscopy.

Before FOB, full blood count, platelet count, and prothrombin time were performed, as was $\frac{\overrightarrow{0}}{0}$ arterial blood gas estimation if there was a clinical $\frac{0}{0}$ suggestion of hypoxaemia. Those patients with a $\frac{\bar{m}}{\mathrm{~m}}$ platelet count below 60000 received transfusions $\overparen{\Phi}$ of six units of fresh donor platelets immediately before the procedure. All patients received कs supplemental oxygen via a single prong nasal $\vec{\circ}$ catheter except for two patients who were ven- $\overrightarrow{\vec{\omega}}$ tilated electively during bronchoscopy because of $\stackrel{\sigma}{\omega}$ very severe arterial hypoxaemia $\left(\mathrm{PO}_{2} 4-5 \mathrm{kPa}\right)$.

Fibreoptic bronchoscopy was carried out with $\underset{\omega}{\omega}$ either an Olympus BF-B2 or BF-B3 bronchoscope. of The patients fasted for at least four hours before the procedure and were given a premedication with omnopon and either scopolamine or atropine $\frac{\text { ? }}{3}$ one hour before bronchoscopy. In thrombocyto- $\vec{c}$ penic patients the premedication was given intravenously at the time of the procedure. The bronchoscope was passed transnasally into the trachea after lignocaine administration into the $\overrightarrow{0}$ nose and oropharynx. ${ }^{16}$ Once the trachea had been $\stackrel{\circ}{\circ}$ entered, a trap was inserted into the suction line to collect any secretions or washings, and the bronchoscope was advanced further into the tracheobronchial tree with administration of $2 \%$ lignocaine to prevent and control coughing. In the $\stackrel{\otimes}{\otimes}$ two patients who were ventilated, the bronchoscope was passed directly into the trachea via an $\overrightarrow{0}$ endotracheal tube through a connector that provides an airtight seal. ${ }^{17}$

A visual examination of the bronchial tree was made in each case and any endobronchial lesions biopsied. If no endobronchial lesion was visible, samples were taken from the affected area under $:$ single plane fluoroscopic control. Where appro- 3 . priate a Medi-Tech sheathed cytology brush ${ }^{18}$ was inserted into the affected area of the lung, and cell debris and secretion collected and placed $\mathrm{O}$ immediately on to bacteriology plates for aerobic and anaerobic culture, fungal and mycobacterial o culture. The tip of the brush was cut off and incubated in thioglycolate for anaerobic $\sigma$ organisms.

Alveolar washings were performed with 50-100 స్ $\mathrm{ml}$ of sterile saline which were instilled into a 0 segmental bronchus on inspiration and aspirated during expiration. The aspirate was sent for bac- $\frac{2}{D}$ teriological, mycological, virological, and cyto- $\stackrel{\infty}{+}$ logical examination. A standard cytology brush 0 (BC-1B) was inserted and smears made for ZeihlNeelson staining, Grocott staining, and cytological $\overparen{\mathbb{D}}$ examination. Multiple transbronchial biopsies were $\mathbb{\mathbb { D }}$ taken from the affected areas of the lung using biopsy forceps and employing a standard technique. ${ }^{19} 20$ The closed forceps were guided to the 
appropriate area with the aid of single plane fluoroscopy as lateral screening facilities were not available. The forceps were then retracted approximately $2 \mathrm{~cm}$, opened, and gently advanced until moderate resistance was encountered or the forceps were within 1-2 cm of the pleura. The forceps were closed and withdrawn with the biopsy sample while the tip of the bronchoscope was left wedged in place in the segmental bronchus to prevent possible haemorrhage by tamponade. Although different areas of the lung were biopsied in diffuse lesions, all the biopsies were carried out on the same side to avoid the possible complication of bilateral pneumothoraces. The alveolar washings and smears sent to the cytology department were stained by standard Papanicolaou and Romanowsky techniques. Further smears were made from the alveolar washings for Grocott silver staining to look for Pneumocystis carinii and fungi. Samples sent to the bacteriology department were plated out on blood agar (anaerobic and aerobic culture), MacConkey, chocolate agar, Sabouraud's medium, and a Lowenstein Jensen slope.

\section{Results}

The results are reported with reference to the type of radiological lesion. The underlying conditions with the corresponding radiographic appearances are listed in table 1 . A total of 55 bronchoscopies was performed in 42 patients, of whom 32 received only one FOB. In seven patients FOB was carried out on two occasions: in three of these a diagnosis of endobronchial Hodgkin's disease had been established at the first bronchoscopy, and the second was to assess new symptoms or response to treatment. One patient developed two separate

Table 1 Underlying diagnosis, chest radiographic abnormalities, and $F O B$ diagnostic yield rate

\begin{tabular}{|c|c|c|c|c|c|}
\hline \multirow[t]{2}{*}{ Underlying condition } & \multirow{2}{*}{$\begin{array}{l}\text { Number } \\
\text { of } \\
\text { patients }\end{array}$} & \multicolumn{4}{|c|}{ Chest radiographic abnormalities } \\
\hline & & Diffuse & Focal & Local & Hilar \\
\hline $\begin{array}{l}\text { Hodgkin's disease } \\
\text { Lymphoma } \\
\text { Acute myeloid leukaemia } \\
\text { Chronic myeloid } \\
\text { leukaemia } \\
\text { Chronic lymphocytic } \\
\text { leukaemia } \\
\text { Acute lymphocytic } \\
\text { leukaemia } \\
\text { Acute undifferentiated } \\
\text { leukaemia }\end{array}$ & $\begin{array}{r}21 \\
9 \\
6 \\
1 \\
3\end{array}$ & $\begin{array}{l}3 \\
5 \\
5 \\
1\end{array}$ & $\begin{array}{l}7 \\
2 \\
1\end{array}$ & 8 & $\begin{array}{l}3 \\
2\end{array}$ \\
\hline Total & 42 & 18 & 11 & 8 & 5 \\
\hline $\begin{array}{l}\text { Number of specific } \\
\text { diagnoses from FOB }\end{array}$ & & 14 & 7 & 2 & 3 \\
\hline $\begin{array}{l}\% \text { positive yield from } \\
\text { FOB }\end{array}$ & & $77 \cdot 8$ & $63 \cdot 6$ & 25 & 60 \\
\hline
\end{tabular}

pulmonary lesions widely separated in time. In the remainder, two FOBs were required to establish the diagnosis. A further group of three patients, all with diffuse lung disease, had three bronchoscopic procedures in an entsavour to make a diagnosis.

These patients had several high risk factors. Most were systemically very sick, but despite this no mortality resulted from the procedures. Twentytwo procedures were complicated by thrombocytopenia of less than 60000 platelets per $\mathrm{mm}^{3}$ and neutropenia. Two had minor haemorrhages of less than $50 \mathrm{ml}$ at the time of procedure. In one other patient haemorrhage occurred at the time of the procedure and continued for 36 hours to a total volume of approx $200 \mathrm{ml}$. Arterial hypoxaemia $\left(\mathrm{PO}_{2}<8 \mathrm{kPa}\right)$ was present in virtually all patients with diffuse radiographic changes and in one case with a focal lesion. The majority tolerated the procedure well.

\section{DIFFUSE RADIOGRAPHIC LESIONS}

Eighteen patients had diffuse lung shadowing (table 1). The diagnoses obtained are listed in table 2. In 11 patients one FOB was performed and a positive diagnosis was obtained in nine $(81.8 \%)$. In four patients FOB was performed on two occasions. Of this group three patients had diffuse lung shadowing on both occasions, with little time elapsing between bronchoscopies. In the remaining case there had been a change from a

Table 2 Diagnosis obtained by FOB with associated chest radiographic abnormalities

\begin{tabular}{|c|c|c|c|c|c|}
\hline \multirow[t]{2}{*}{ Diagnosis } & \multirow{2}{*}{$\begin{array}{l}\text { Total } \\
\text { number }\end{array}$} & \multicolumn{4}{|c|}{ Chest radiographic abnormalities } \\
\hline & & Diffuse & Focal & Local & Hilar \\
\hline \multirow{2}{*}{\multicolumn{6}{|c|}{$\begin{array}{l}\text { Infiltration with } \\
\text { underlying disease }\end{array}$}} \\
\hline & & & & \\
\hline endobronchial & 2 & & 2 & & \\
\hline parenchymal & 2 & 2 & & & \\
\hline \multicolumn{6}{|l|}{ Hodgkin's disease } \\
\hline endobronchial & 2 & & & & 2 \\
\hline parenchymal & 3 & 2 & 1 & & \\
\hline \multicolumn{6}{|l|}{ Carcinoma } \\
\hline Squamous cell & 2 & 1 & 1 & & \\
\hline Adenocarcinoma & 1 & & & 1 & \\
\hline \multirow{2}{*}{\multicolumn{6}{|c|}{$\begin{array}{l}\text { Infections } \\
\quad \text { Haemophilus influenzi }\end{array}$}} \\
\hline Haemophilus influenzi & & & & & \\
\hline $\begin{array}{l}\text { pneumonia } \\
\text { Staphylococcus aureus }\end{array}$ & 1 & & 1 & & \\
\hline pneumonia & 1 & 1 & & & \\
\hline Aspergillus fumigatus & 1 & 1 & & & \\
\hline Pulmonary haemorrhage & 3 & 3 & & & \\
\hline Cytotoxic lung & 4 & 4 & & & \\
\hline \multicolumn{6}{|l|}{ Others } \\
\hline Lipoid pneumonia & 2 & & 1 & 1 & \\
\hline Asthma & 1 & & & & 1 \\
\hline $\begin{array}{l}\text { Lymphomatoid } \\
\text { granulomatosis }\end{array}$ & 1 & & 1 & & \\
\hline Total & 26 & 14 & 7 & 2 & 3 \\
\hline
\end{tabular}


localised type of lesion to a more diffuse lung involvement over a longer period. A positive diagnosis was achieved in three of these four, in one of whom two different causes were established on the two occasions. In a further three patients FOB was performed on three occasions within a relatively short period of time, but no evidence of infection or pulmonary involvement by the disease process was found. One of these patients, who had thrombocytopenia, probably had a pulmonary haemorrhage as numerous haemosiderin-laden macrophages were seen, and one patient was found at postmortem to have had pulmonary oedema secondary to a large aortic thrombus.

Overall a positive diagnosis was made in 14 $(78 \%)$ of these patients, and infection as a cause was almost certainly excluded in a further three. The results of FOB influenced treatment in 11 patients $(61 \%)$ but mortality remained very high, 14 patients $(77 \cdot 8 \%)$ dying within three months, most within the first two weeks. Of the four patients who survived for between eight and 24 months, treatment was undoubtedly influenced by FOB.

\section{FOCAL RADIOGRAPHIC CHANGES}

Eleven patients had segmental or lobar involvements of the lung which were designated as "focal" pulmonary lesions. A positive diagnosis was made in seven cases $(63.6 \%)$. The diagnoses obtained are shown in table 2 . In one patient the initial diagnosis of Hodgkin's disease was changed to lymphomatoid granulomatosis on the basis of the histological samples obtained by FOB. Where a diagnosis was not achieved three patients probably had pulmonary involvement with Hodgkin's disease as judged by their subsequent clinical course. In the other undiagnosed patient histology showed some of the macrophages to have formed foreign-body-type giant cells, the significance of which remained uncertain. There was, however, no evidence of an infective aetiology for the pulmonary lesion. Where a diagnosis was obtained treatment was influenced in each case, with improvement in four patients. A patient with squamous cell carcinoma received conservative therapy. Two other patients, in whom a diagnosis was not made, died and permission for necropsy was refused. Survival times for the remainder varied between 10 and 28 months.

LOCAL RADIOGRAPHIC CHANGES

Eight patients had small pulmonary lesions of less than $3 \mathrm{~cm}$ diameter. In one of these patients the lesion progressed undiagnosed to diffuse involve- ment for which a further FOB was performed. $\frac{\text { की }}{\frac{7}{0}}$ This established pulmonary infiltration with $\frac{0}{0}$ Hodgkin's disease, the latter result being included $\overline{\frac{\sigma}{\omega}}$ in the group with diffuse changes. A positive diag- $\frac{\vec{\Phi}}{\alpha}$ nosis was made in two patients (table 2). In this group all patients had Hodgkin's disease and the $s$ undiagnosed lesion probably constituted local in- $\overrightarrow{0}$ volvement with this condition. Most of these $\overrightarrow{-}$ patients subsequently received chemotherapy with $\vec{\sigma}$ resolution of the lesions and all have remained alive for between nine and 29 months.

\section{HILAR RADIOGRAPHIC CHANGES}

Five patients had a prominent hilum or definite $\vec{\bullet}$ hilar abnormality. A diagnosis of Hodgkin's disease was made in two by FOB. One patient had ${ }_{\vec{C}}$ a prominent hilum and was dyspnoeic. No endo-ฏ bronchial lesion was seen but biopsy of the bronchial mucosa showed the changes of asthma and this fitted in with the clinical picture: she re- $\vec{c}$ sponded to normal therapy for asthma. In the twoo cases where diagnoses were not obtained the hilar. changes were probably the results of glandular in-o volvement by lymphoma, and these patients later received chemotherapy and improved.

\section{Discussion}

Although there have been several studies on the value of the various invasive techniques used in the diagnosis of pulmonary disease, only a few. have looked at the role of FOB in immunocompromised patients who are both at high risk and have a wide range of possible causes for their ${ }_{-x}^{x}$ lesions. Some of these studies have confined them-3. selves to the use of one of the available proceduresin that may be performed at bronchoscopy-for example, alveolar lavage ${ }^{9}$ or bronchial brushings. ${ }^{8}$ Both Cunningham et $a l^{21}$ and Matthay et $a l^{160}$ have shown that the diagnostic success rate rise when bronchial brushing is combined with trans bronchial biopsy (TBB) and so in each instances we used all the available procedures which includeh alveolar lavage, selected cultures with a sheatheof brush, standard bronchial brushing, and transbronchial biopsy.

The analysis of our results is based on the radio은 graphic appearance for two reasons. First, theres appeared to be a difference in the aetiology and outcome between the groups. Secondly, there was a considerable disparity in the diagnostic yield rated achieved in the various groups (table 1). The mose striking difference was the low yield in patients with local pulmonary lesions. We felt that this was mainly because we only had single plane 
fluoroscopy to help in the positioning of the brush or biopsy forceps, which could thus be either in front or behind the lesion in the AP view. With a rotating table, so that lateral screening could be achieved, we believe our diagnostic yield rate could have been greater than the $25 \%$ we obtained. However, it is probable that an additional factor is also responsible as the yield rate in small peripherally situated bronchial carcinoma in our hands is $62 \%$ (unpublished data). It is probable that this infiltration was mainly peribronchial, ${ }^{3}$ therefore few neoplastic cells would be shed on alveolar lavage or bronchial brushing in contrast to endobronchial lesions. Thus a well-positioned transbronchial biopsy is required to obtain the necessary diagnostic material.

In contrast, a high yield rate was obtained in those patients with diffuse or focal lesions. Even in those patients in whom a specific diagnosis was not achieved the procedure was useful in virtually excluding infection as a cause of the pulmonary lesion. If nonspecific interstitial pneumonitis is included as a diagnosis our yield rate for diffuse lesions would rise from $14 / 18(78 \%)$ to $16 / 18$ $(89 \%)$, and for focal lesions from $7 / 11(64 \%)$ to $8 / 11(73 \%)$.

Our results compare well with other published series on immuno-compromised patients. Cunningham et $a l^{21}$ obtained a specific diagnosis in $48 \%$ of transbronchial biopsy procedures combined with bronchial brushing and $65 \%$ of percutaneous trephine lung biopsies. When a diagnosis of nonspecific interstitial pneumonitis was included the yield rate rose to $74 \%$ and $82 \%$ respectively. Matthay et al ${ }^{16}$ obtained a specific diagnosis in 10 out of $11(91 \%)$ focal lesions and 11 out of 14 $(78.6 \%)$ diffuse lesions using combined bronchial brushing and TBB. A considerably lower yield is obtained if bronchial brushing alone is employed, varying from $28 \%{ }^{8}$ to $63 \% .^{16}$

In the initial phase of the study we repeated the procedure for a second or even third time in those patients where TBB revealed nonspecific changes. We found these repeat procedures unhelpful in that they did not provide further information, and the patients' later progress did not suggest that any treatable condition had been missed. However repeat TBB was of value in three cases where the tissue was histologically normal in the first instance indicating that it had not come from the appropriate lesion.

Several transbronchial biopsies showed nonspecific histology with no evidence of any infective aetiology. In three patients a dense fibrinous exudate was found with evidence of damage to the alveoli. This may have resulted from cytotoxic therapy but might represent an organising pneumonic process in a patient without adequate granulocytic function and hence without the normal methods of removing the intra-alveolar exudate.

The diagnosis of pulmonary haemorrhage has been made on the basis of numerous haemosiderinladen macrophages found in the alveolar lavage, at a time when there had been no overt haemorrhage or other cause of bleeding apart from thrombocytopenia. The diagnosis was made in three patients all of whom had diffuse radiographic shadowing and thrombocytopenia. The diagnosis was confirmed by necropsy findings in one patient and supported by histology from transbronchial biopsy in another. Recently Drew et $a^{9}{ }^{9}$ have suggested that occult pulmonary haemorrhage may be more common in thrombocytopenic immunocompromised patients than has been recognised hitherto, and they reported nine out of 12 consecutive patients who had this problem. In our series we found evidence of pulmonary haemorrhage in only three patients out of 15 who were thrombocytopenic.

One of the striking features in our series is the relative lack of bacterial or other opportunistic pathogens as aetiological agents for the pumonary lesions. This is in sharp contrast to other studies where the most common diagnosis was infection, whether caused by bacteria, Pneumocystis carinii, fungi, or more rarely viruses. ${ }^{810111621}$ Most of these studies are from the USA where fungal infections are in general more frequent than in Britain. In addition the difference may be the result of some selection in the patients coming to FOB because a policy had been adopted of treating with broad spectrum antibiotics all pyrexial patients with focal or diffuse pulmonary lesions. Those in whom resolution or improvement of their lesions did not occur were referred for bronchoscopy. Several patients who were suspected clinically of having Pneumocystis carinii were treated with high doses of co-trimoxazole with subsequent resolution of the pulmonary lesions. The series was thus biased away from simple infective causes. Probably as a consequence of this we found that most of our information was derived from histology of the transbronchial biopsy specimens and cytological examination of bronchial brushings, and that little additional information was forthcoming from selective cultures with a sheathed brush. Alveolar lavage was helpful more for cytological examination than for bacteriology. Even when simple bacteria were isolated there were often difficulties of interpretation because of 
the problems of contamination of the bronchoscope by oropharyngeal organisms, and this was not avoided entirely by the use of sheathed brushes which may also become contaminated during their passage down the lumen of the bronchoscope.

Haemorrhage as a result of transbronchial biopsy was not a problem in our series even in those patients who were thrombocytopenic. This was presumably attributable to our policy of giving fresh donor platelet transfusions to all patients who had a platelet count of less than 60000 . However, none of our patients was significantly uraemic which may also have contributed to the lower incidence of haemorrhage, as it is known that this predisposes to bleeding. ${ }^{2122}$

The only other complications found in this series were three pneumothoraces, one of which occurred in a patient who was being ventilated because of severe hypoxaemia. In the other two patients the pneumothorax was asymptomatic but one received intercostal drainage in view of the degree of collapse of the lung.

Thus FOB and TBB appear to be relatively safe techniques in the investigation of immunocompromised patients with pulmonary complications, despite the presence of thrombocytopenia and arterial hypoxaemia, providing platelet transfusions and supplemental oxygen are administered.

In general, other invasive procedures have provided yield rates that are no better and often worse than FOB, but in addition are associated with a greater morbidity. Thus open lung biopsy in this situation has given specific diagnoses of $79 \%{ }^{11}$ and $71 \%{ }^{12}$ but with the discomfort and morbidity associated with operation. Needle aspiration biopsies give generally lower yields of between $60 \%$ and $73 \%$, and pulmonary haemorrhage has been reported to occur in $8 \%,,^{11}$ with an $8-30 \%$ incidence of pneumothoraces. ${ }^{10}{ }^{23}$ Cutting needle biopsies may give yields of up to $75 \%{ }^{10}$ but not only have a high incidence of pneumothoraces, but in this type of patient carry a real risk of haemorrhage which is difficult to controlGreenman et $a l^{10}$ reported a $15 \%$ incidence of haemorrhage with this technique. Trephine drill biopsies may provide good samples of lung for histology and Cunningham et $a l^{21}$ were able to obtain a specific diagnosis in $65 \%$ of cases, but this procedure had a $60 \%$ incidence of pneumothorax and a $17 \%$ incidence of haemorrhage.

Fibreoptic bronchoscopy offers the advantages of more satisfactory control of any haemorrhage which occurs by the local instillation of adrenaline solutions and by using the "wedge" technique as described by Zavala ${ }^{14}$ to act as a tamponade. There has been no recorded fatality from TBB in this situation, although this has occurred with the use $\frac{\text { ? }}{\circ}$ of the cutting needle biopsy technique. ${ }^{10}$ The inci- $\frac{\bar{\sigma}}{\bar{c}}$ dence of pneumothoraces is much lower than with $\vec{\Phi}$ the use of percutaneous techniques, and it is 0 possible to sample parenchyma from a variety of $\approx$ sites as well as collect bronchial secretions and $\vec{\circ}$ diagnose endobronchial lesions.

Although a specific diagnosis was made in a high $\vec{\rho}$ percentage of patients with diffuse disease, the $\stackrel{F}{\vec{F}}$ clinical outcome remained poor with only four out $\times$ of 18 surviving in this group. One of these patients 0 had infiltration with lymphoma and one with Hodgkin's disease; both responded to chemo- $\overrightarrow{0}$ therapy. The other two had cytotoxic lung. In $\frac{1}{2}$ general, immuno-compromised patients with dif- fuse pulmonary disease are in a precarious state, $\stackrel{c}{\stackrel{c}{ }}$ so that a poor prognosis is not unexpected, but the circumstances may be particularly unfavourable in our group because of the bias away from simple $\vec{\bullet}$

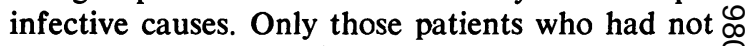
responded to antibiotics were selected for bron-. choscopy and thus included in this series. Although many of the patients proved to be unresponsive to further treatment, a diagnosis was still helpful $\frac{\partial}{0}$ in providing a more rational basis for management.

There was a more favourable outcome for those $\stackrel{\mathbb{2}}{\square}$ patients with other than diffuse lesions. There was $\overrightarrow{\overrightarrow{0}}$ a good diagnostic yield in the group with focal 3 lesions, and this unquestionably influenced further management. Eight out of the 11 patients in this? group remain alive. All patients with hilar abnormalities had Hodgkin's disease or non-Hodgkin's 음 lymphoma, and most received chemotherapy or $\tilde{x}$ radiotherapy with subsequent resolution. All are $\frac{0}{3}$ still alive.

We achieved a poor diagnostic success rate in patients with local lesions. All these patients had Hodgkin's disease but with little evidence of $\frac{9}{\square}$ activity elsewhere. Many of the patients were $\frac{?}{0}$ asymptomatic and the lesions only came to notice on routine chest film. The disease, therefore, ap- $N$ peared to be in a more benign phase than in those patients with diffuse or focal changes, and this is $N$ reflected in the fact that all patients in this group $\omega$ remain alive. Most of the patients received further chemotherapy on the grounds that the likely diag- $\stackrel{\varrho}{C}$ nosis was lung infiltration with Hodgkin's disease, $\stackrel{\mathcal{D}}{\mathcal{D}}$ and indeed the lesions resolved or regressed on this :treatment. Despite the poor yield rate from FOB $\frac{0}{0}$ in this category, this procedure may reveal other $\stackrel{\mathbb{D}}{\mathbb{D}}$ factors such as the unexpected diagnosis of adeno- $\frac{\stackrel{\rho}{\mathbb{D}}}{\mathbb{Q}}$

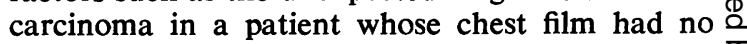
distinguishing features from others in the group.

We conclude that FOB has a useful role in the 
investigation of pulmonary lesions in the immunocompromised patient. In general the procedure is well tolerated and the morbidity is low, conferring an advantage over many of the alternative invasive techniques. The yield rate varies according to the type of pulmonary lesions, being most successful in patients with diffuse or focal radiographic changes where it compares very favourably with other techniques. Despite the fact that our yield with local lesions was poor we still believe that FOB should remain the invasive procedure of first choice whatever the type of pulmonary lesion. Although the final outcome is frequently poor, a proportion of patients are unquestionably helped by a definitive diagnosis, and the management of the remainder is put on a more rational basis.

We should like to thank Professor JS Malpas and $\mathrm{Dr} \mathrm{A}$ Lister for their help and co-operation throughout the study.

\section{References}

1 Williams DM, Krick JA, Remington JS. Pulmonary infection in the compromised host. $\mathrm{Am}$ Rev Respir Dis 1976; 114:359-94, 593-627.

2 Ellman P, Bowdler AJ. Pulmonary manifestations of Hodgkin's disease. Br J Dis Chest 1960; 54:5971.

3 Macdonald JB. Lung involvement in Hodgkin's disease. Thorax 1977; 32:664-7.

4 Bodey GP, Powell RD, Hersch EM, Yeterian A, Freireich EJ. Pulmonary complications of acute leukemia. Cancer 1966; 19:781-93.

5 Nathan DJ, Sanders M. Manifestations of acute leukemia in the parenchyma of the lung. $N$ Engl $J$ Med 1955; 252:797-801.

6 Canellos GP, DeVita VT, Arseneau JC, WhangPeng J, Johnson REC. Second malignancies complicating Hodgkin's disease in remission. Lancet 1975; 1:947-9.

7 Rosenow FC. The spectrum of drug-induced pulmonary disease. Ann Intern Med 1972; 77:977-91.

8 Finley $\mathrm{R}$, Kieff $\mathrm{E}$, Thomsen $\mathrm{S}$ et al. Bronchial brushing in the diagnosis of pulmonary disease in patients at risk for opportunistic infection. $\mathrm{Am}$ Rev Respir Dis 1974; 104:379-87.
9 Drew WL, Finley TN, Golde DW. Diagnostic lavage and occult pulmonary hemorrhage in thrombocytopenic immunocompromised patients. Am Rev Respir Dis 1977; 116:215-21.

10 Bandt PD, Blank N, Castellino RA. Needle diagnosis of pneumonitis. Value in high-risk patients. JAMA 1972; 220:1578-80.

11 Greenman RL, Goodall PT, King D. Lung biopsy in immunocompromised hosts. Am J Med 1975; 59:488-96.

12 Leight GS, Michaelis LL. Open lung biopsy for the diagnosis of acute, diffuse pulmonary infiltrates in the immunosuppressed patient. Chest 1978; 73:477-82.

13 Sackner MA. Bronchofiberscopy. Am Rev Respir Dis 1975; 111:62-88.

14 Zavala DC. Diagnostic fiberoptic bronchoscopy: techniques and results of biopsy in 600 patients. Chest 1975; 68:12-19.

15 Knight RK, Clarke SW. Fibreoptic bronchoscopy: applications. Practitioner 1977; 218:28694.

16 Matthay RA, Farmer WC, Odero D. Diagnostic fibreoptic bronchoscopy in the immunocompromised host with pulmonary infiltrates. Thorax 1977; 32:539-45.

17 Knight RK. Fibreoptic bronchoscopy in intensive care. Br Med J 1976; 2:1564-5.

18 Aisner J, Kvols LK, Sickles EA, Schimpff SC, Wiernik PH. Transtracheal selective bronchial brushing for pulmonary infiltrates in patients with cancer. Chest 1976; 69:367-71.

19 Levin DC, Wicks AB, Ellis JH Jr. Transbronchial lung biopsy via the fiberoptic bronchoscope. $\mathrm{Am}$ Rev Respir Dis 1974; 110:4-12.

20 Joyner LR, Scheinhorn DJ. Transbronchial forceps lung biopsy through the fiberoptic bronchoscope. Chest 1975; 67:532-5.

21 Cunningham JH, Zavala DC, Corry JJ, Keim LW. Trephine air drill, bronchial brush, and fiberoptic transbronchial lung biopsies in immunosuppressed patients. Am Rev Respir Dis 1977; 115:213-20.

22 Kazatchkine M, Sultan Y, Caen JP, Bariety J. Bleeding in renal failure: a possible cause. $\mathrm{Br}$ Med J 1976; 2:612-5.

23 Dahlgren $S$, Nordenström $D$. Transthoracic needle biopsy. Chicago: Year Book Medical Publishers, 1966.

24 Zavala DC. Pulmonary hemorrhage in fiberoptic transbronchial biopsy. Chest 1976; 70:584-8. 